\title{
NEED OF VOCATIONAL HIGH SCHOOL BASED ON LOCAL POTENTIAL FOR REGIONAL DEVELOPMENT IN CILACAP DISTRICT
}

\author{
Widi Lesmana \\ Universitas Negeri Yogyakarta \\ Pardjono \\ Universitas Negeri Yogyakarta
}

\begin{abstract}
This study intends to: (1) describe regional development programs, (2) determine local potential, (3) describe the level of alignment of the current spectrum of expertise and make a spectrum of expertise Vocational High Schools based local potential in the Cilacap District. Descriptive research was used in this study. The research site was carried out in the Cilacap Regency area. The data sources used are secondary data sources, namely the use of data through official documents such as the Rencana Tata Ruang Wilayah (RTRW), Rencana Pembangunan Jangka Menengah Daerah (RPJMD), Cilacap Regency in the 2017 Figures and the Cilacap Regency Economic Census in 2016. Analysis of official documents are used to achieve the research objectives. The validity of the documents carried out is the provision of a wet stamp from the related agency. The results of this study include: (1) the development program planned for the development of local potential in the Cilacap Regency area is listed on the 4th mission of regional development, namely "developing an economy that relies on local and regional potential"; (2) existing local potential includes the manufacturing sector; agriculture, forestry and fisheries; wholesale and retail trade, car and motorcycle repair; and the construction sector; (3) the level of alignment of the expertise spectrum of SMK with local potential in corridor 1 amounting to $35.7 \%$; corridor 2 of $28.6 \%$; corridor 3 is $43 \%$ while corridor 4 is $28.5 \%$; areas of expertise that are in harmony with local potential include: Technology and Engineering; Agribusiness and Agrotechnology; Maritime; Business and management. The needs of local potential vocational schools are 36 schools, 465 classes and 887 teachers. It is necessary to close and increase the areas of expertise, expertise programs and expertise competencies to be in harmony with local potential in the Cilacap Regency and its surrounding areas.
\end{abstract}

Keywords: vocational school based local potential, regional development, cilacap region

Permalink: http://dx.doi.org/10.21831/jpv.v9i1.21701

Contact Widi Lesmana widilesmana19.2017@student.uny.ac.id Universitas Negeri Yogyakarta, Jl. Colombo No. 1, Depok, Sleman 55281, Yogyakarta, Indonesia 


\section{INTRODUCTION}

Economic inequality between regions is the background for why regional-based potential optimization is interesting to analyze. Cilacap Regency GRDP is ranked 2 as the biggest contributor to GDP in 2016 after Semarang, which is 9.22 percent. The economic potential of Cilacap Regency is mainly in the category of Manufacturing Industry. The role of the Manufacturing Industry category is 64.09 percent of the GRDP of Cilacap Regency, which is the largest in the Food and Beverage Industry subcategory. There are similarities in the potential of the region owned to the 24 subdistricts, because of the conditions of nature, culture, education and so forth. It is necessary to divide the development sub-region to facilitate the categorization of the potential of the area owned. The administrative map of the Cilacap Regency area can be seen in the following Figure 1.

Based on the Education and Culture Statistics Data Center, the number of vocational high school students in Cilacap Regency in $2017 / 2018$ is 38,534. This amount is an accumulation of 65 existing schools. The potential of the workforce in the vocational secondary education sector in Cilacap Regency in 2017/ 2018 is 13,362 graduates who are ready to work according to their respective fields of expertise (Direktorat Pembinaan Sekolah Menengah Kejuruan, 2018). The number of graduates indicates that every year Cilacap has prospective skilled workers who are ready to work and advance the region. Clarke \& Winch (2007, p. 9) says that vocational education is limited to preparing the population for life in the workforce. Whereas according to Gunderson (2004, p. 3), vocational education includes courses and programs at various levels of education for certain careers in the field of work. According to Billet (2011, p. 2), vocational education as "education for employment" namely the ability to carry out work that is in accordance with the field of expertise must be owned by students. Vocational education in an international context, often referred to as TVET (Technical and Vocational Education and Training). TVET is related to the acquisition of knowledge and skills for the world of work (Gough, 2010, p. 1). Vocational education involves the preparation of people for the world of work, that is, preparing them to "make a living" by the nearest and distant communities (Thomas, 2005, p. 626). In line with that, Rauner (2009, p. 1451) states that vocational education is different from all types of education institutionalized in schools and universities.

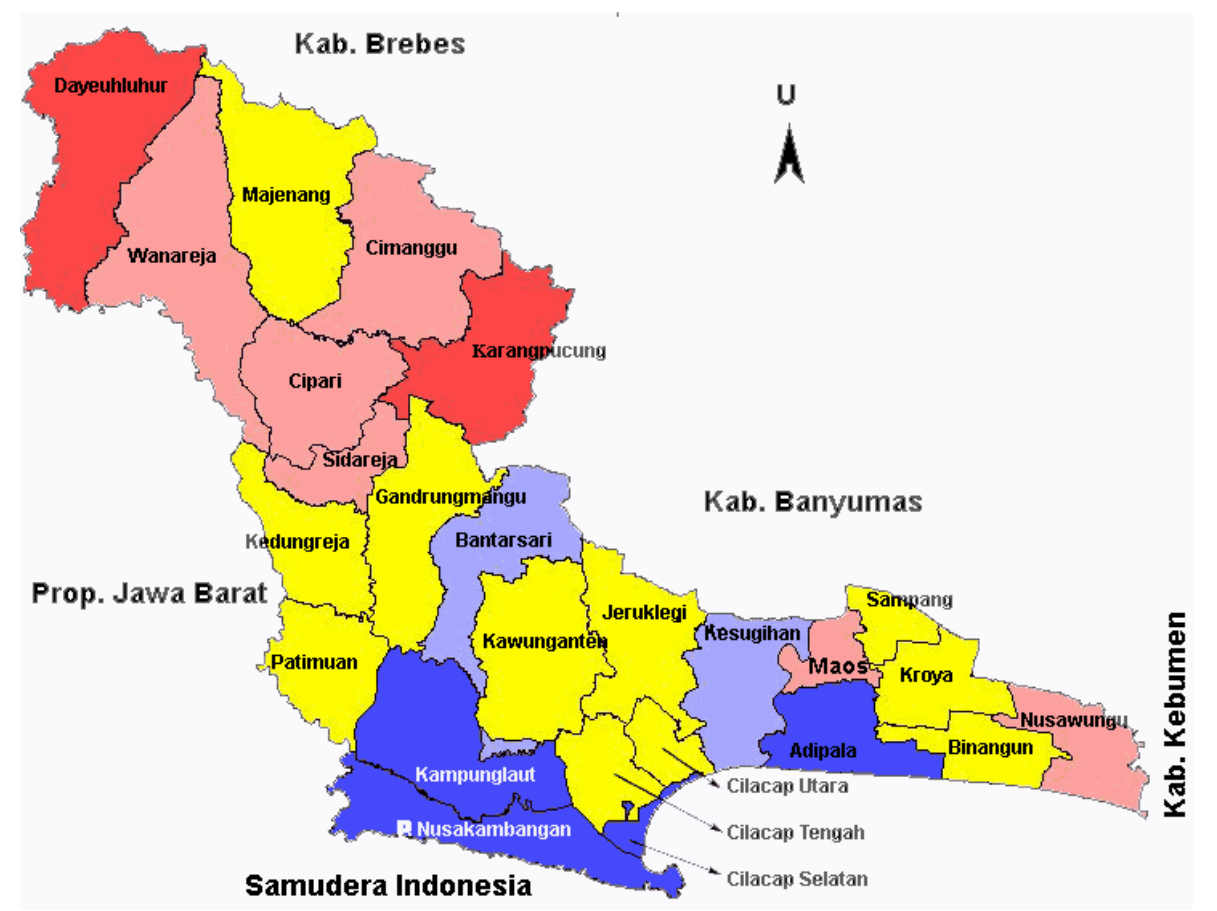

Figure 1. Map of Cilacap Regency Area (Source: BPS Documents) 
Understanding of the type of command or assignment of a work related to the development of knowledge in an education is called vocational education (Sudira, 2016, p. 9). Pavlova (2009) said the objectives of the tradition of vocational education include:

Traditionally, direct preparation for work was the main goal of vocational education. It was perceived as providing specific training that was reproductive and based on teachers'instruction, with the itention to develop understanding of a particular industry, comprising the specific skills or tricks of the trade. Students' motivation was seen to be engendered by the economic benefit to them, in the future. Competency-based training was chosen by most governments in western societis as a model for vocational education (Pavlova, 2009, p. 7).

The main purpose of vocational education if viewed traditionally is the direct preparation of prospective workers to work. In line with Pavlova (2009) \& Rojewski (2009) says the tradition of vocational education prepares skilled workers with high abilities that are subject to employers. But the open unemployment rate of vocational school graduates is actually very high compared to other education levels. This condition is caused by a mismatch of fields of expertise possessed by the needs of the workforce, and graduates without skills who can face a disruptive era. Renewing the vision of vocational education has an impact on changing educational goals, teaching and learning activities, learning tools and teaching based on world-class vocational education (Pavlova \& Munjanganja, 2009, p. 80; Cheng, 2006, p. 25). According to Sudira (2015, p. 2) skills to deal with the 21 st century include LIS 5C, namely Learning-Innovation Skill (LIS): Critical thinking and problem solving; Communications and Collaboration; Creativity and Innovation. Whereas according to Trilling \& Fadel $(2009$, p. 8$)$ the new skills needed by the world of work today are complex communication and expert thinking. Skills including complex communication are communications and collaboration while critical thinking and problem solving fall into the category of expert thinking skills. While creativity and innovation skills become supporters of the application skills of imagination and discovery. Vocational educa- tion as an institution for preparing human resources (HR) for the world of work needs to pay attention to issues and renewal of the education substance (Sawyer, 2012; Littleton, Taylor, \& Eteläpelto, 2012; Taylor, 2012).

According to Maley, Worley, \& Dent (2009), career graduates can have a quality lifestyle with the provision of longer learning programs. In addition to learning programs, Maley, et al (2009) also mentions the main factor for achieving student success is through an approach by developing professional teachers who utilize local technology and culture. Whereas according to Abdullah, Hussin, Shonubi, Ghazali, \& Talib (2018, p. 71) there is a significant relationship between self knowledge, job exploration, and career decision making. In line with Maley, et al (2009) \& Hee (2014) says that in the learning process, students tend to like the deep learning approach of age and gender groups. Whereas according to Paimin, Hadgraft, Prpic, \& Alias (2011), learning strategies do not have a direct relationship with the intention of learning unless mediated by motives for learning. According to Cournoyer \& Deschenaux (2017), decision making by graduates is characterized by: (1) socio-professional and economic decline; (2) recognize yourself, personally and socially; (3) theoretical and practical values; (4) utilizing supporting conditions; and (5) closeness reconciliation. Wagiran (2008) shows that the top ten skills expected by industry include aspects of honesty, work ethic, responsibility, discipline, application of the principles of safety, initiative, creativity, cooperation, adjustment, selfconfidence, and tolerance.

\section{RESEARCH METHOD}

The type of research used in this study is descriptive research with a document analysis approach. The procedure of the research carried out was by collecting official documents, then carried out an analysis to produce regional development programs, local potential, the spectrum of expertise of the existing vocational secondary schools and those which should be based on local potential. The analysis to determine the local potential which is a superior and potential sector is using LQ (Local Quotient) analysis. The instruments in this study were researchers, flashdisks, internet networks, notebooks, and cameras. The area of Cilacap 
Regency is divided into 4 corridors for subdevelopment areas, which consist of each corridor totaling 6 sub-districts. Corridor 1 consists of six sub-districts, namely Dayehluhur, Wanareja, Majenang, Cimanggu, Cipari, and Karangpucung. Corridor 2 consists of six sub-districts, namely Sidareja, Gandrungmangu, Kawunganten, Kedungreja, Bantarsari, and Patimuan. Corridor 3 consists of six subdistricts, namely Adipala, Binangun, Kampung Laut, South Cilacap, Central Cilacap, and Cilacap Utara. Corridor 4 consists of six subdistricts, namely Nusawungu, Jeruk Legi, Kesugihan, Maos, Sampang, and Kroya. The grouping is considered based on the map of the administrative area and existing local potential characters.

\section{RESULT AND DISCUSSION}

\section{Results}

\section{Regional Development Program}

Based on the results of data analysis conducted, the results of the research were obtained to solve the research questions. The results of the first study were a description of the regional development program owned by Cilacap District within a period of 5 years (see Table 1)

\section{Local Potential}

The results of the second study are related to local potential which is the leading and potential sector in each corridor of the development area (see Tables 2 and 3 ).

Table 1. Direction of Regional Development of Cilacap Regency

\begin{tabular}{cl}
\hline Year & \multicolumn{1}{c}{ Direction of Development Policy } \\
\hline 2018 & $\begin{array}{l}\text { Improving the quality of basic education services in the realization of schools Minimum service } \\
\text { standards are focused on providing quality education infrastructure (quality of classrooms, teacher's } \\
\text { room, library and laboratory quality) and cultural character }\end{array}$ \\
2019 & $\begin{array}{l}\text { Improving the quality of basic health facilities and infrastructures and referencing in the realization of } \\
\text { healthy communities is focused on realizing quality basic service facilities, quality referral facilities and } \\
\text { clean and healthy lifestyles, providing social rehabilitation to people with social problems (PMKS), } \\
\text { integration of gender mainstreaming (PUG) ) and the Child Rights Mainstreaming Strategy (PUHA) }\end{array}$ \\
2020 & $\begin{array}{l}\text { Economic development and infrastructure in the framework of the region strengthens the development } \\
\text { of peripheral and border areas focused on increasing access to tourism villages, innovation villages and } \\
\text { infrastructure for economic development in the community. Democratic economic development is } \\
\text { focused on improving the quality of micro-enterprises, cooperatives and enhancing traditional markets }\end{array}$ \\
2021 & $\begin{array}{l}\text { Improving the quality of regional infrastructure in Strengthening the development of peripheral and } \\
\text { border areas focused on road repair, drainage, irrigation, waste management }\end{array}$ \\
2022 Improving the quality of regional infrastructure to achieve universal access (slum, sanitation and \\
drinking water) is focused on fulfilling public housing, drinking water, sanitation and public space
\end{tabular}

(Source: Data Analysis Results, 2019)

Table 2. Local Superior Sectors

\begin{tabular}{cll}
\hline $\begin{array}{c}\text { Development } \\
\text { Area }\end{array}$ & \multicolumn{1}{c}{ Business field } & \multicolumn{1}{c}{ Criteria } \\
\hline Corridor 1 & Agriculture, Forestry, Fisheries & Featured Sector (Base) \\
& Big Trade and Retail, Car and Motorcycle Repair & Featured Sector (Base) \\
Corridor 2 & Agriculture, Forestry, Fisheries & Featured Sector (Base) \\
Corridor 3 & Manufacturing Industry & Featured Sector (Base) \\
& Big Trade and Retail, Car and Motorcycle Repair & Featured Sector (Base) \\
& Construction & Featured Sector (Base) \\
Corridor 4 & Manufacturing Industry & Featured Sector (Base) \\
& Agriculture, Forestry, Fisheries & Featured Sector (Base) \\
& Big Trade and Retail, Car and Motorcycle Repair & Featured Sector (Base) \\
& Construction & Featured Sector (Base) \\
\hline
\end{tabular}

(Source: Data Analysis Results, 2019) 
Table 3. Potential Sectors in each Corridor

\begin{tabular}{cll}
\hline $\begin{array}{c}\text { Development } \\
\text { Area }\end{array}$ & \multicolumn{1}{c}{ Business field } & \multicolumn{1}{c}{ Criteria } \\
\hline Corridor 1 & Manufacturing Industry & Potential Sector (Non Base) \\
& Construction & Potential Sector (Non Base) \\
Corridor 2 & Manufacturing Industry & Potential Sector (Non Base) \\
& Agriculture, Forestry, Fisheries & Potential Sector (Non Base) \\
& Big Trade and Retail, Car and Motorcycle Repair & Potential Sector (Non Base) \\
& Construction & Potential Sector (Non Base) \\
Corridor 3 & Agriculture, Forestry, Fisheries & Potential Sector (Non Base) \\
Corridor 4 & - & \\
\hline
\end{tabular}

(Source: Data Analysis Results, 2019)

\section{Level of Alignment of the Expert Spectrum}

Alignment levels were analyzed based on the spectrum of expertise in each corridor of the development area. The results of the third study are related to the existing level of SMK spectrum alignment with local potential (see Table 4, 5, 6, 7).

Table 4. Level of Alignment of Existing Vocational Expertise Spectrum with Local Potential in Corridor 1

\begin{tabular}{|c|c|c|c|c|}
\hline No. & Areas of expertise & Expertise Program & Skill Competence & $\begin{array}{l}\text { Level } \\
\text { Alignment }\end{array}$ \\
\hline \multirow[t]{6}{*}{1} & \multirow{6}{*}{$\begin{array}{l}\text { Technology and } \\
\text { Engineering }\end{array}$} & $\begin{array}{l}\text { Technology and } \\
\text { Construction }\end{array}$ & $\begin{array}{l}\text { Construction and Property } \\
\text { Business }\end{array}$ & Conformable \\
\hline & & Electricity Technique & $\begin{array}{l}\text { Electric Power Installation } \\
\text { Techniques }\end{array}$ & Misaligned \\
\hline & & \multirow{3}{*}{$\begin{array}{l}\text { Mechanical Engineering } \\
\text { Automotive Engineering }\end{array}$} & Machining Techniques & Misaligned \\
\hline & & & $\begin{array}{l}\text { Automotive Light Vehicle } \\
\text { Engineering }\end{array}$ & Conformable \\
\hline & & & $\begin{array}{l}\text { Motorcycle Engineering and } \\
\text { Business }\end{array}$ & Conformable \\
\hline & & Chemical Engineering & chemical analysis & Misaligned \\
\hline \multirow[t]{2}{*}{2} & \multirow{2}{*}{$\begin{array}{l}\text { Information and } \\
\text { communication } \\
\text { technology }\end{array}$} & \multirow{2}{*}{$\begin{array}{l}\text { Computer Engineering } \\
\text { and Informatics }\end{array}$} & Multimedia & Misaligned \\
\hline & & & $\begin{array}{l}\text { Computer and Network } \\
\text { Engineering }\end{array}$ & Misaligned \\
\hline \multirow[t]{2}{*}{3} & \multirow[t]{2}{*}{$\begin{array}{l}\text { Agribusiness and } \\
\text { Agro-technology }\end{array}$} & Agribusiness Crop & $\begin{array}{l}\text { Agribusiness Food Crops and } \\
\text { Horticulture }\end{array}$ & Conformable \\
\hline & & Agribusiness Livestock & Agribusiness Poultry & Conformable \\
\hline \multirow[t]{4}{*}{4} & \multirow[t]{4}{*}{$\begin{array}{l}\text { Business and } \\
\text { management }\end{array}$} & Office management & $\begin{array}{l}\text { Office automation and } \\
\text { Governance }\end{array}$ & Misaligned \\
\hline & & \multirow{3}{*}{$\begin{array}{l}\text { Business and Marketing } \\
\text { Accounting and finance }\end{array}$} & Online business and marketing & Conformable \\
\hline & & & $\begin{array}{l}\text { Accounting and finance } \\
\text { institutions }\end{array}$ & Misaligned \\
\hline & & & Syariah banking & Misaligned \\
\hline \multirow[t]{2}{*}{5} & \multirow[t]{2}{*}{ Tourism } & Kulinary & Cullinary art & Misaligned \\
\hline & & Fashion & Fashion & Misaligned \\
\hline \multirow[t]{2}{*}{6} & \multirow{2}{*}{$\begin{array}{l}\text { Health and social } \\
\text { work }\end{array}$} & \multirow[t]{2}{*}{ Pharmacy } & Pharmaceutical Industry & Misaligned \\
\hline & & & $\begin{array}{l}\text { Clinical and Community } \\
\text { Pharmacy }\end{array}$ & Misaligned \\
\hline \multirow[t]{2}{*}{7} & \multirow{2}{*}{$\begin{array}{l}\text { Arts and Creative } \\
\text { Industries }\end{array}$} & Alignment (Total Align/I & Animation & \multirow{2}{*}{$\begin{array}{c}\text { Misaligned } \\
35.7 \% \\
\end{array}$} \\
\hline & & Alignment (Total Align/I & al x 100\%) & \\
\hline
\end{tabular}

(Source: Data Analysis Results, 2019) 
Table 5. Spectrum's Expertise Level Vocational Existing Alignment with Local Potential Corridor 2

\begin{tabular}{|c|c|c|c|c|}
\hline No. & $\begin{array}{l}\text { Areas of } \\
\text { expertise }\end{array}$ & Expertise Program & Skill Competency & $\begin{array}{c}\text { Level } \\
\text { Alignment }\end{array}$ \\
\hline \multirow[t]{6}{*}{1} & Technology and & Mechanical Power & Mechanical Power Installation & Misaligned \\
\hline & Engineering & Automotive Engineering & Automotive Light Vehicle Engineering & Conformable \\
\hline & & & Techniques and Business Motorcycle & Misaligned \\
\hline & & Mechanical Engineering & Mechanical Machining & Misaligned \\
\hline & & & Welding techniques & Misaligned \\
\hline & & Electrical engineering & Mechanical Audio Video & Misaligned \\
\hline \multirow[t]{2}{*}{2} & Information and & Computer Engineering & Multimedia & Misaligned \\
\hline & $\begin{array}{l}\text { communication } \\
\text { technology }\end{array}$ & $\begin{array}{l}\text { and Information } \\
\text { Technology }\end{array}$ & Computer and Network Engineering & Misaligned \\
\hline 3 & $\begin{array}{l}\text { Agribusiness and } \\
\text { Agro-technology }\end{array}$ & Agribusiness Crop & $\begin{array}{l}\text { Agribusiness Food Crops and } \\
\text { Horticulture }\end{array}$ & Conformable \\
\hline \multirow[t]{3}{*}{4} & Business and & Office management & Office automation and Governance & Misaligned \\
\hline & management & Business and Marketing & Online business and marketing & Conformable \\
\hline & & Accounting and finance & Accounting and finance institutions & Misaligned \\
\hline 6 & $\begin{array}{l}\text { Health and Social } \\
\text { Work }\end{array}$ & Pharmacy & Clinical and Community Pharmacy & Misaligned \\
\hline 7 & Maritime & Cruise Fishing Vessels & Teknika Fishing Vessels & conformable \\
\hline \multirow[t]{2}{*}{8} & $\begin{array}{l}\text { Arts and Creative } \\
\text { Industries }\end{array}$ & Art & Animation & Misaligned \\
\hline & & \multicolumn{2}{|c|}{ Level Alignment (Total Align/Ideal x 100\%) } & $28.6 \%$ \\
\hline
\end{tabular}

(Source: Data Analysis, 2019)

Table 6. Spectrum's expertise level vocational Existing Alignment with Local Potential Corridor 3

\begin{tabular}{|c|c|c|c|c|}
\hline No. & $\begin{array}{l}\text { Areas of } \\
\text { expertise }\end{array}$ & Expertise Program & Skill competency & $\begin{array}{l}\text { Level } \\
\text { Alignment }\end{array}$ \\
\hline 1 & $\begin{array}{l}\text { Technology and } \\
\text { Engineering }\end{array}$ & $\begin{array}{l}\text { Mechanical Power } \\
\text { Technology Construction } \\
\text { and Property } \\
\text { Automotive Engineering } \\
\text { Mechanical Engineering }\end{array}$ & $\begin{array}{l}\text { Mechanical Power Installation } \\
\text { Mechanical Power Plant } \\
\text { Business Construction and Property } \\
\text { Design and Building Information Modeling } \\
\text { Automotive Light Vehicle Engineering } \\
\text { Techniques and Business Motorcycle } \\
\text { Mechanical Machining } \\
\text { Welding techniques }\end{array}$ & $\begin{array}{l}\text { Misaligned } \\
\text { Misaligned } \\
\text { conformable } \\
\text { conformable } \\
\text { conformable } \\
\text { Misaligned } \\
\text { Misaligned } \\
\text { Misaligned }\end{array}$ \\
\hline 2 & $\begin{array}{l}\text { Information and } \\
\text { communication } \\
\text { technology }\end{array}$ & $\begin{array}{l}\text { Computer Engineering } \\
\text { and Information } \\
\text { Technology }\end{array}$ & $\begin{array}{l}\text { Multimedia } \\
\text { Computer and Network Engineering }\end{array}$ & $\begin{array}{l}\text { Misaligned } \\
\text { Misaligned }\end{array}$ \\
\hline 3 & $\begin{array}{l}\text { Agribusiness } \\
\text { and } \\
\text { Agrotechnology }\end{array}$ & Agribusiness Crop & Agribusiness Food Crops and Horticulture & conformable \\
\hline 4 & $\begin{array}{l}\text { Business and } \\
\text { management }\end{array}$ & $\begin{array}{l}\text { Office management } \\
\text { Business and Marketing } \\
\text { Accounting and finance }\end{array}$ & $\begin{array}{l}\text { Office automation and Governance } \\
\text { Online business and marketing } \\
\text { Accounting and finance institutions } \\
\text { Syariah banking }\end{array}$ & $\begin{array}{c}\text { Misaligned } \\
\text { conformable } \\
\text { Misaligned } \\
\text { Misaligned }\end{array}$ \\
\hline 5 & $\begin{array}{l}\text { Health and } \\
\text { Social Work } \\
\text { maritime }\end{array}$ & Cruise Fishing Vessels & Pharmaceutical Industry & Misaligned \\
\hline 7 & $\begin{array}{l}\text { Energy and } \\
\text { Mining }\end{array}$ & Oil Technic & Mechanical Oil, Gas and Petrochemical & Misaligned \\
\hline 8 & Tourism & $\begin{array}{l}\text { Hospitality and Tourism } \\
\text { Services } \\
\text { Culinary } \\
\text { Fashion } \\
\text { Level Alignment (Total A }\end{array}$ & $\begin{array}{l}\text { Hospitality } \\
\text { Cullinary art } \\
\text { Fashion } \\
\text { lign/Ideal x 100\%) }\end{array}$ & $\begin{array}{l}\text { Misaligned } \\
\text { Misaligned } \\
\text { Misaligned } \\
\quad 43 \%\end{array}$ \\
\hline
\end{tabular}

(Source: Data Analysis, 2019) 
Table 7. Spectrum's expertise level vocational Existing Alignment with Local Potential in Corridor 4

\begin{tabular}{|c|c|c|c|c|}
\hline No. & $\begin{array}{l}\text { Areas of } \\
\text { expertise }\end{array}$ & Expertise Program & Skill competency & $\begin{array}{c}\text { Level } \\
\text { Alignment }\end{array}$ \\
\hline \multirow[t]{9}{*}{1} & \multirow{9}{*}{$\begin{array}{l}\text { Technology and } \\
\text { Engineering }\end{array}$} & \multirow[t]{2}{*}{ Mechanical Power } & Mechanical Power Installation & Misaligned \\
\hline & & & Mechanical Power Plant & Misaligned \\
\hline & & \multirow[t]{5}{*}{ Automotive Engineering } & Automotive Light Vehicle & conformable \\
\hline & & & Engineering & \\
\hline & & & Techniques and Business & Misaligned \\
\hline & & & Motorcycle & \\
\hline & & & Mechanical Ototronik & Misaligned \\
\hline & & Mechanical Engineering & Mechanical Machining & Misaligned \\
\hline & & Electrical engineering & Mechanical Audio Video & Misaligned \\
\hline \multirow[t]{3}{*}{2} & \multirow{3}{*}{$\begin{array}{l}\text { Information and } \\
\text { communication } \\
\text { technology }\end{array}$} & \multirow{3}{*}{$\begin{array}{l}\text { Computer Engineering } \\
\text { and Information } \\
\text { Technology }\end{array}$} & Multimedia & Misaligned \\
\hline & & & Computer and Network & Misalioned \\
\hline & & & Engineering & \\
\hline \multirow[t]{4}{*}{3} & \multirow{4}{*}{$\begin{array}{l}\text { Business and } \\
\text { management }\end{array}$} & Office management & Office automation and Governance & Misaligned \\
\hline & & Business and Marketing & Online business and marketing & conformable \\
\hline & & Accounting and finance & Accounting and finance institutions & Misaligned \\
\hline & & & Syariah banking & Misaligned \\
\hline \multirow[t]{2}{*}{4} & \multirow[t]{2}{*}{ maritime } & \multirow[t]{2}{*}{ Cruise Fishing Vessels } & Nautical Fishing Vessels & conformable \\
\hline & & & Teknika Fishing Vessels & conformable \\
\hline \multirow[t]{4}{*}{5} & \multirow[t]{4}{*}{ Tourism } & $\begin{array}{l}\text { Hospitality and Tourism } \\
\text { Services }\end{array}$ & hospitality & Misaligned \\
\hline & & Culinary & Cullinary art & Misaligned \\
\hline & & Fashion & Fashion & Misaligned \\
\hline & & \multicolumn{2}{|c|}{ Level Alignment (Total Align/Ideal x 100\%) } & $28.5 \%$ \\
\hline
\end{tabular}

(Source: Data Analysis, 2019)

\section{SMK needs Based Local Potential}

The results of the fourth study is SMK needs based on local potential. Consists of a spectrum of vocational skills based on local potential, the need for teachers, school and grade (see Table $8,9,10,11,12)$.

Table 8. Spectrum Needs Vocational Skills-Based Local Potential Corridor 1

\begin{tabular}{|c|c|c|c|c|c|}
\hline \multirow[t]{2}{*}{ No. } & \multirow{2}{*}{$\begin{array}{l}\text { Areas of } \\
\text { Expertise }\end{array}$} & \multirow[t]{2}{*}{ Expertise Program } & \multirow[t]{2}{*}{ Skill competency } & \multicolumn{2}{|c|}{$\begin{array}{l}\text { Education } \\
\text { Programs }\end{array}$} \\
\hline & & & & $3 \mathrm{Th}$ & $4 \mathrm{Th}$ \\
\hline \multirow[t]{7}{*}{1} & Agribusiness and & Agribusiness & Agribusiness Agricultural & $\sqrt{ }$ & \\
\hline & Agrotechnology & $\begin{array}{l}\text { Agricultural Product } \\
\text { Processing }\end{array}$ & Product Processing & & \\
\hline & & Agribusiness Crop & $\begin{array}{l}\text { Agribusiness Food Crops and } \\
\text { Horticulture }\end{array}$ & $\sqrt{ }$ & \\
\hline & & & Agribusiness Crops & $\sqrt{ }$ & \\
\hline & & Agribusiness Livestock & Agribusiness Ruminant & $\sqrt{ }$ & \\
\hline & & & Agribusiness Poultry & $\sqrt{ }$ & \\
\hline & & Forestry & $\begin{array}{l}\text { Forest Products Production } \\
\text { Technology }\end{array}$ & $\sqrt{ }$ & \\
\hline 2 & Maritime & Fishery & $\begin{array}{l}\text { Freshwater Fishery } \\
\text { Agribusiness }\end{array}$ & $\sqrt{ }$ & \\
\hline 3 & $\begin{array}{l}\text { Technology and } \\
\text { Engineering }\end{array}$ & Automotive Engineering & $\begin{array}{l}\text { Automotive Light Vehicle } \\
\text { Engineering }\end{array}$ & $\sqrt{ }$ & \\
\hline 4 & $\begin{array}{l}\text { Business and } \\
\text { management }\end{array}$ & Business and Marketing & $\begin{array}{l}\text { Online Business and } \\
\text { Marketing }\end{array}$ & $\sqrt{ }$ & \\
\hline & & & Retail & $\sqrt{ }$ & \\
\hline
\end{tabular}

(Source: Data Analysis, 2019) 
Table 9. Spectrum Needs Vocational Skills-Based Local Potential Corridor 2

\begin{tabular}{|c|c|c|c|c|c|}
\hline \multirow[t]{2}{*}{ No. } & \multirow[t]{2}{*}{ Areas of Expertise } & \multirow[t]{2}{*}{ Expertise Program } & \multirow[t]{2}{*}{ Skill competency } & \multicolumn{2}{|c|}{$\begin{array}{l}\text { Education } \\
\text { Programs } \\
\end{array}$} \\
\hline & & & & $3 \mathrm{Th}$ & $4 \mathrm{Th}$ \\
\hline \multirow[t]{5}{*}{1} & Agribusiness and & Agribusiness Agricultural & $\begin{array}{l}\text { Agribusiness Agricultural Product } \\
\text { Processing }\end{array}$ & $\sqrt{ }$ & \\
\hline & & Agribusiness Crop & $\begin{array}{l}\text { Agribusiness Food Crops and } \\
\text { Horticulture } \\
\text { Agribusiness Crops }\end{array}$ & $\begin{array}{l}\sqrt{ } \\
\sqrt{ }\end{array}$ & \\
\hline & & Agribusiness Livestock & Agribusiness Ruminant & $\sqrt{ }$ & \\
\hline & & & Agribusiness Poultry & $\sqrt{ }$ & \\
\hline & & Forestry & $\begin{array}{l}\text { Forest Products Production } \\
\text { Technology }\end{array}$ & $\sqrt{ }$ & \\
\hline 2 & maritime & Fishery & Freshwater Fishery Agribusiness & $\sqrt{ }$ & \\
\hline
\end{tabular}

(Source: Data Analysis, 2019)

Table 10. Spectrum Needs Vocational Skills-Based Local Potential Corridor 3

\begin{tabular}{|c|c|c|c|c|}
\hline \multirow[t]{2}{*}{ No. } & \multirow{2}{*}{$\begin{array}{l}\text { Areas of } \\
\text { expertise }\end{array}$} & \multirow[t]{2}{*}{ Expertise Program } & \multirow[t]{2}{*}{ Skill competency } & $\begin{array}{l}\text { Education } \\
\text { Programs }\end{array}$ \\
\hline & & & & $3 \mathrm{Th} \quad 4 \mathrm{Th}$ \\
\hline \multirow[t]{4}{*}{1} & $\begin{array}{l}\text { Technology and } \\
\text { Engineering }\end{array}$ & $\begin{array}{l}\text { Technology Construction } \\
\text { and Property }\end{array}$ & $\begin{array}{l}\text { Building Construction, Sanitation and } \\
\text { Maintenance }\end{array}$ & $\sqrt{ }$ \\
\hline & & & $\begin{array}{l}\text { Road Construction, Irrigation and } \\
\text { Bridges }\end{array}$ & $\sqrt{ }$ \\
\hline & & & $\begin{array}{l}\text { Design and Building Information } \\
\text { Modeling }\end{array}$ & $\sqrt{ }$ \\
\hline & & Automotive Engineering & Automotive Light Vehicle Engineering & $\sqrt{ }$ \\
\hline \multirow[t]{2}{*}{2} & Business and & Business and Marketing & Online Business and Marketing & $\sqrt{ }$ \\
\hline & management & & Retail & $\sqrt{ }$ \\
\hline
\end{tabular}

(Source: Data Analysis, 2019)

Table 11. Spectrum Needs Vocational Skills-Based Local Potential in Corridor 4

\begin{tabular}{|c|c|c|c|c|c|}
\hline \multirow[t]{2}{*}{ No. } & \multirow[t]{2}{*}{ Areas of expertise } & \multirow[t]{2}{*}{ Expertise Program } & \multirow[t]{2}{*}{ Skill competency } & \multicolumn{2}{|c|}{$\begin{array}{l}\text { Education } \\
\text { Programs }\end{array}$} \\
\hline & & & & $3 \mathrm{Th}$ & $4 \mathrm{Th}$ \\
\hline \multirow[t]{7}{*}{1} & Agribusiness and & Agribusiness & Agribusiness Agricultural Product & $\sqrt{ }$ & \\
\hline & Agrotechnology & Agricultural Product & Processing & & \\
\hline & & $\begin{array}{l}\text { Processing } \\
\text { agribusiness Crop }\end{array}$ & $\begin{array}{l}\text { Agribusiness Food Crops and } \\
\text { Horticulture }\end{array}$ & $\sqrt{ }$ & \\
\hline & & & Agribusiness Crops & $\sqrt{ }$ & \\
\hline & & agribusiness Livestock & Agribusiness Ruminant & $\sqrt{ }$ & \\
\hline & & & Agribusiness Poultry & $\sqrt{ }$ & \\
\hline & & Forestry & $\begin{array}{l}\text { Forest Products Production } \\
\text { Technology }\end{array}$ & $\sqrt{ }$ & \\
\hline \multirow[t]{3}{*}{2} & Maritime & Cruise Fishing Vessels & Nautical Fishing Vessels & $\sqrt{ }$ & \\
\hline & & Fishery & Freshwater Fishery Agribusiness & $\sqrt{ }$ & \\
\hline & & & $\begin{array}{l}\text { Agribusiness Fisheries Brackish } \\
\text { Water and Marine }\end{array}$ & $\sqrt{ }$ & \\
\hline \multirow[t]{4}{*}{3} & $\begin{array}{l}\text { Technology and } \\
\text { Engineering }\end{array}$ & $\begin{array}{l}\text { Technology Construction } \\
\text { and Property }\end{array}$ & $\begin{array}{l}\text { Building Construction, Sanitation } \\
\text { and Maintenance }\end{array}$ & $\sqrt{ }$ & \\
\hline & & & $\begin{array}{l}\text { Road Construction, Irrigation and } \\
\text { Bridges }\end{array}$ & $\sqrt{ }$ & \\
\hline & & & $\begin{array}{l}\text { Design and Building Information } \\
\text { Modeling }\end{array}$ & $\sqrt{ }$ & \\
\hline & & Automotive Engineering & $\begin{array}{l}\text { Automotive Light Vehicle } \\
\text { Engineering }\end{array}$ & $\sqrt{ }$ & \\
\hline \multirow[t]{2}{*}{4} & $\begin{array}{l}\text { Business and } \\
\text { management }\end{array}$ & Business and Marketing & Online Business and Marketing & $\sqrt{ }$ & \\
\hline & management & & 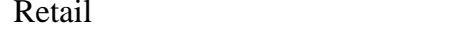 & & \\
\hline
\end{tabular}

(Source: Data Analysis, 2019) 
Table 12. Vocational needs in each Corridor Development Area

\begin{tabular}{cccc}
\hline $\begin{array}{c}\text { Territory } \\
\text { Development }\end{array}$ & $\begin{array}{c}\text { School } \\
\text { needs }\end{array}$ & $\begin{array}{c}\text { Classroom } \\
\text { needs }\end{array}$ & $\begin{array}{c}\text { Teacher } \\
\text { needs }\end{array}$ \\
\hline corridor 1 & 9 & 108 & 228 \\
corridor 2 & 17 & 202 & 337 \\
corridor 3 & 4 & 76 & 137 \\
corridor 4 & 6 & 79 & 185 \\
total & 36 & 465 & 887 \\
\hline
\end{tabular}

(Source: Data Analysis, 2019)

\section{Discussion}

The high rate of unemployment of graduates of vocational school does not mean that the skills possessed incompetent, but many factors that influence such a mismatch of expertise with the needs of the workforce. Academic qualification is not a tool to ensure graduates obtain employment (Mazwin, 2006). Regional development programs aligned with CMS as a labor provider to establish the region can have an impact on the decline in the unemployment rate. Companies across the world are considering the graduates and their readiness to work (NICHE, 2009). Moreover, the quality of students as interns greatly influencing skills and personality after being labor graduate (Jingzhou, Yanjun, Jingru, Lihong, FeiZhu, Xinyu, \& Jinmeng, 2018). The ability to build human relationships, the ability to utilize the information, the ability to plan for the future, and the ability to make decisions is a goal that should be given priority in the planning of vocational courses to produce graduates who are productive (Morita, 2018, p. 70). Sukardi (2011) said that knowledge or skill if done regularly and continuously, students/graduates will master the knowledge or the material thoroughly. According to Abdurrahman (2016), a program developed in vocational skills tend to be based on the needs of the potential of the area, just a little effort vocational graduates who can be absorbed by the local industry because most industries are still using traditional methods in knowledge as well as the production process. One cause of the graduates are not absorbed optimally is because middle-age population can not have that can facilitate the vocational school, by Mirza (2008) is not yet even education, especially in terms of quantity / number of vocational schools affect the amount of skilled labor produced, so the industry will use the labor outside the area. Kilpatrick (2004) ar- gues that the education and training institutions have an important role in the development of the regions or the key to the interaction of the public and the government in regional development. The growth of an economy that occurred in the region will have an impact not only on economic growth in the region, but also in other parts of the economy (Setiawan, 2006). Kilpatrick (2004) argues that the education and training institutions have an important role in the development of the regions or the key to the interaction of the public and the government in regional development. The growth of an economy that occurred in the region will have an impact not only on economic growth in the region, but also in other parts of the economy (Setiawan, 2006). Kilpatrick (2004) argues that the education and training institutions have an important role in the development of the regions or the key to the interaction of the public and the government in regional development. The growth of an economy that occurred in the region will have an impact not only on economic growth in the region, but also in other parts of the economy (Setiawan, 2006).

Educational development based on local potentials rightly done extensive and systematic studies to produce proper planning target. In this study, conducted analiss local potential of the flagship, determine the spectrum of vocational skills based on local potentials, analyze the potential for population and employment. These variables should be mutually supportive vocational development. According Wagiran (2010), there are four main focus on the indepth study, namely; (a) an analysis of the potential and resources, (b) the preparation of some concepts of neighborhood-based education development (area), (c) short-term programming, medium, long, (d) human resource planning and supporting facilities.

Alignment level vocational skills spectrum conditions that exist today with local potential is still low. At one level alignment corridor is only $35.7 \%$, in the corridor 2 was $28.6 \%$ and the third corridor of $43 \%$ while $28.5 \% 4$ corridor. There are a lot of competency skills that should exist but not in any CMS. So that these conditions allow to make one of the reasons the unemployment rate is still higher vocational graduates. CMS needs to maximize the potential of the population can be used as new ways to increase the number of skilled labor. 
Potential resources owned by Indonesia both natural and human potential should be managed better in improving the local economy. One solution for the government in improving the economic condition is to make the most of the potential of nature and the number of people as workers. Not all communities in all regions in Indonesia can receive education up to secondary school level, the gross enrollment rate indicates that there are still many people aged 16-19 years who did not receive education up to upper secondary level. The role of government was needed to reduce the school dropout rate.

Vocational Secondary School is a secondary educational institutions that can be used as a tool of labor scorer reliable. However, graduates of vocational far only capable of being a labor used for its own interests and the company in place of work (a foreign company) that would benefit outsiders. It takes a shift in the orientation of vocational graduates so that the potential of skilled labor can be utilized to promote the region and the country. So that economic inequality in each area can be reduced.

\section{CONCLUSION}

Planning vocational training based on local potentials can be done by analyzing the potential of the area to determine the economic sector which is the base and non-base, making a reference spectrum of expertise that is consistent with the potential of the area and to identify the level of alignment of the existing spectrum of expertise. In addition analysis of population aged 16-18 years who have not attained secondary education is needed, because in order to plan the needs of vocational and high school with a 70:30 ratio to facilitate the population. SMK needs based on local potential which has been obtained based on the research results are expected to assist the government in developing the area, reduce unemployment and boost the economy of the community, and create a new orientation for vocational school graduates to be able to work on building areas within maximize the potential of nature. The new orientation can be a solution for the government in preparing a skilled workforce.

\section{REFERENCES}

Abdullah, N., Hussin, N., Shonubi, O. A., Ghazali, S. R., \& Talib, M. A. (2018).
Career decision-making competence, self-knowledge, and occupational exploration: a model for university students. Journal Of Technical Education And Training, 10(1).

Abdurrahman, A. (2016). Analisis kebutuhan dan penyediaanpengembangan SMK berbasis potensi daerah Kabupaten Bantaeng (Doctoral Dissertation, Universitas Negeri Makassar).

Badan Perencanaan Pembangunan Nasional. (2018). Proyeksi penduduk di Indonesia tahun 2010-2035.

Badan Pusat Statistik Nasional. (2018). Angka pengangguran terbuka. Retrieved 1 September 2018 from http://www.bps.go.id/.

Badan Pusat Statistik Nasional. (2018). Jumlah angkatan kerja. Retrieved 1 September from 2018 http://www.bps.go.id/.

Billet, S. (2011). Vocational education purposes, traditions and prospects. London: Springer Science+Business Media.

Cheng, Y. C. (2006). New paradigm for reengineering education: Globalization, localization and individualization (Vol. 6). Springer Science \& Business Media.

Clarke, L., \& Winch, C. (2007) Vocational education: international approacher, development, and system. New York: 270 Madesun Avenue, 2007.

Cournoyer, L., \& Deschenaux, F. (2017). Decision-making rationales among Quebec VET student aged 25 and older. International Journal for Research in Vocational Education and Training (IJRVET), 4(3), 226-248.

Direktorat Pembinaan Sekolah Menengah Kejuruan. (2018). Data pokok SMK. Retrieved from http://datapokok.ditpsmk.net/

Gough, S. (2010). Technical and vocational education and learning: an investmentbased approach. Great Britain: the MPG Books Group, Bodmin and King's Lynn, 2010.

Gunderson, M. M. A. (2004). Study of the 
influence vocational education has on students' ultimate academic success. (Disertasi doktor, University of Central Florida, 2004). ProQuest Information and Learning Company, UMI Number: 3162094, 2004.

Hee, O. C. (2014). A study on the learning approach of the Malaysian adult students. Journal of Technical Education and Training, 6(2).

Jingzhou, P., Yanjun, G., Jingru, W., Lihong, H., FeiZhu, Xinyu, F., \& Jinmeng, Y. (2018). The interplay of proactive personality and internship quality in Chinese university graduates' job search success: The role of career adaptability. Journal of Vocational Behavior, 109, 14-26.

Kilpatrick, S. (2004). Education and Training Institutions: building social capital for regional development. Research and Learning in Regional Australia, University of Tasmania, Australia.

Littleton, K., Taylor, S., \& Eteläpelto, A. (2012). Special issue introduction: Creativity and creative work in contemporary working contexts. Vocations and Learning, 5(1), 1-4

Maley, M., Worley, P., \& Dent, J. (2009). Using rural and remote settings in the undergraduate medical curriculum: AMEE Guide No. 47. Medical Teacher, 31(11), 969-983.

Mazwin, N.A. (2006). Too much job-hopping bad for career. Retrieved May 15, 2008, from http://www.

starjobs.com/news/story.asp?file=/2006/ 3/15/starjobs/13663628\&sec=starjobs

Mirza, I. (2008). Pengembangan sekolah kejuruan berbasis potensi pengembangan wilayah di Kabupaten Brebes (Doctoral dissertation, Universitas Diponegoro).

Morita, T., Yamamoto, K., \& Managi, S. (2018). The relationship between schoolbased career education and subsequent incomes: Empirical evidence from Japan. Economic Analysis and Policy, $58,70-87$.

NICHE. (2009). Higher Education in the
Learning Society (The Dearing Report), London, 1997, (paragraph 4.22). Retrieved February 10, 2009, from bei.leeds.ac.uk/Partners/NICHE/.

Paimin, A. N., Hadgraft, R., Prpic, K., \& Alias, M. (2011). Learning strategy, motive and intention: predicting academic performance of engineering undergraduates. In Proceedings of the IETEC'11 Conference.

Pavlova, M. (2009). Technology and vocational education for sustainable development empowering individuals for the future. Queensland: Springer Science Business Media B.V.

Pavlova, M., \& Munjanganja, L. E. (2009). Section 1 changing workplace requirements: implications for education. International Handbook of Education for the Changing World of Work: Bridging Academic and Vocational Learning, 1(80).

Rauner, F.(2009). Overview: TVET research. international handbook of education for the changing world of work. Germany: Springer Science+Business Media B.V.

Rojewski, J. W. (2009). A conceptual framework for technical and vocational education and training. In International handbook of education for the changing world of work (pp. 19-39). Springer, Dordrecht.

Sawyer, K. (2012). Extending sociocultural theory to group creativity. Vocations and Learning, 5(1), 59-75.

Setiawan, I. D. M. D. (2006). Peranan sektor unggulan terhadap pertumbuhan ekonomi daerah: pendekatan inputoutput multiregional Jawa Timur, Bali, dan Nusa Tenggara Barat. Soca (SocioEconomic Of Agriculturre And Agribusiness).

Sudira, P. (2015). Pengembangan model "Lis$5 \mathrm{c}$ " pada pendidikan teknologi dan kejuruan. Cakrawala Pendidikan, 34(1), $1-11$.

Sudira, P. (2016). TVET abad XXI, filosofi, teori konsep, dan strategi pembelajaran vokasional. Yogyakarta: UNY Press. 
Sukardi, T. (2011). Peran bimbingan kejuruan terhadap pembentukan karakter kerja siswa di Jurusan Mesin SMKN 2 Wonosari. Jurnal Cakrawala Pendidikan, Th. XXX, Edisi Khusus Dies Natalis UNY.

Taylor, S. (2012). The meanings and problems of contemporary creative work. Vocations and Learning, 5(1), 4157.

Thomas, R.M. (2005). Education from an international perspective. encyclopedia of education and human development. New York: M.E. Sharpe, Inc.

Trilling, B., \& Fadel, C. (2009). 21st century skills: Learning for life in our times.
Sanfrancisco: Jossey Bass.

Wagiran. (2008). The importance of developing soft skills in preparing vocational high school graduates. In International Conference on VTE Research and Networking 2008: Nurturing Local VTE Research Efforts: A Response to Global Challenges $7-8$ July 2008 Inna Grand Bali Beach Hotel, Bali, Indonesia.

Wagiran. (2010). Pengembangan pendidikan kejuruan berbasis potensi daerah dan sumberdaya alam dalam mendukung continuing vocational education. In Seminar Internasional ISSN 1907-2066. 